\title{
Modélisation de l'évaporation globale d'un couvert forestier II. - Calibrages et résultats du modèle
}

\author{
P. CHASSAGNEUX, E. CHOISNEL \\ Météorologie nationale, Subdivision d'Agrométéorologie, \\ 2, avenue Rapp, F 75007 Paris
}

\begin{abstract}
Résumé
La seconde partie de cet article présente la comparaison des résultats du modèle avec des mesures neutroniques hebdomadaires du déstockage du sol pour trois années (1976, 1977 et 1979) sous couvert de feuillus (Fagus silvatica L.) et pour une année (1978) sous couvert de résineux (Pseudotsuga menziesii). Les différentes étapes de calibrage du modèle sont énumérées : date de débourrement et date de feuillaison complète pour les feuillus, ajustement des différentes résistances en sćrie, la résistance aérodynamique, les deux résistances de structure et la résistance stomatique. L'étape intermédiairc de calibrage du sous-modèle d'interception montre que l'erreur relative sur la pluic interceptée est de 8 p. 100 en été et de 14 p. 100 en hiver pour le couvert de feuillus.

La comparaison entre le calcul et la mesure du déstockage du sol, dont la réserve utile est fixée à $170 \mathrm{~mm}$, montre une simulation correcte de l'assèchement du sol de mai à juillet au cours de l'année 1976 de sécheresse exceptionnelle. En annéc très pluvieuse (1977) la simulation du déstockage est satisfaisante. L'année 1979, où la phase de débourrement-feuillaison du hêtre a été tardive mais courte, laisse apparaitre une sous-estimation de l'évapotranspiration réelle du couvert forestier.

Un indice de stress hydrique, calculé à partir des donnćes de sortie du modèle est proposé. Le modèle permet également de simuler sur une longue période climatique, à partir des données de la station météorologique de Nancy-Essey, le bilan hydrique d'un couvert de feuillus d'une part et d'un couvert de résineux d'autre part. Sur la période de feuillaison (du 15 mai au 15 octobre) l'évapotranspiration calculée du couvert de feuillus est supérieure de $20 \%$ à celle du couvert de résineux. Malgré une interception des résineux plus importante que celle des feuillus sur cette même période de 5 mois l'évaporation globale (évapotranspiration + interception) du couvert de feuillus reste légèrement supérieure à celle du couvert de résincux.
\end{abstract}

Mots clés : Déstockage du sol, évaporation, évapotranspiration, feuillus, interception, modèle, résineux, résistance.

\section{Introduction}

Dans la première partie de cet article (Chassagneux \& Chorsnel, 1986) nous avons présenté les équations utilisées pour la modélisation de l'évaporation globale du couvert. Nous avons aussi énuméré et décrit les différents paramètres du modèle. Les constantes intervenant dans le calcul de ces paramètres ont été déterminées par ajustement des sorties du modèle à des mesures expérimentales effectuées au C.N.R.F. de Nancy sous couvert de feuillus (Aussenac \& Granier, 1979) et sous couvert de résineux (Aussenac \& Boul.angeat, 1980 et données non publiées). Mais pour ce faire il convenait d'adopter un certain ordre chronologique pour la détermination des 
constantes. Nous avons choisi de calibrer d'abord le sous-modèle «interception », puis le sous-modèle "transpiration de l'arbre" de façon à minimiser l'écart entre le déstockage du sol mesuré et le déstockage du sol calculé par le modèle. La simulation des variables écophysiologiques et les paramètres de régulation du modèle seront d'abord examinés avant de présenter les résultats proprement dits. Enfin les applications possibles d'un tel modèle sont envisagées.

\section{Profils climatiques des années d'expérimentation}

Les tests du modèle pour le peuplement feuillu ont concerné les années 1976, 1977, 1978, 1979 et 1981 et pour le peuplement résineux les années 1978 et 1979. L'analyse statistique des précipitations pour les quatre "saisons météorologiques" (l'hiver de décembre à février, le printemps de mars à mai, l'été de juin à août et l'automne de septembre à novembre), extraite du dossier de statistiques agroclimatiques (1951-1980) de la station de Nancy-Essey indique que les mesures de sondages neutroniques disponibles correspondent à des années très différentes sur le plan climatique et en particulier :

-.- une année très sèche (hiver, printemps et été 1976) ;

- deux années très pluvieuses (les quatre saisons pour les années 1977 et 1979), avec en particulier pour l'année 1979 un hiver et un printemps exceptionnellement pluvieux (maximum pluviométrique pour la période 1951-1980).

\section{Les variables écophysiologiques}

\subsection{Couvert de feuillus}

Au cours de l'année quatre stades phénologiques sont définis pour le hêtre : le débourrement, la date de feuillaison complète. la date de perte des premières feuilles, la date de début de la période défeuillée. Le scius-modèle phénologique utilisé pour simuler l'évolution de l'indice foliaire de l'arbre au printemps a été décrit dans la première partie de cet article (ChASSAGneux \& ChoISnel, 1986 ; NIZINSKI, 1986). Une simulation sur trente-trois années des dates de détiourrement et de feuillaison complète ont donné les résultats suivants pour Nancy:

\begin{tabular}{|c|c|c|}
\hline & Dibourrement & Feuillaison complète \\
\hline Date la plus précoce & 16 mars & 13 avril \\
\hline Premier quintile. & 28 mars & 24 avril \\
\hline Médiane $\ldots \ldots \ldots \ldots \ldots \ldots \ldots$ & 11 avril & 7 mai \\
\hline Quatric̀me quintile & 2 mai & 24 mai \\
\hline Date la plus tardive ..... & 18 mai & 3 juin \\
\hline
\end{tabular}


Ces dates coïncident avec les estimations données par Aussenac (comm. pers.) pour les valeurs moyennes, respectivement 10 avril et 10 mai. On remarque à l'examen de cette statistique une grande variabilité interannuelle possible de ces deux stades phénologiques. Entre ces deux dates se situe la phase d'évolution de l'indice foliaire jusqu'à sa valeur maximale. La figure 1 illustre le décalage de croissance de l'indice foliaire pour deux années très différentes (1976 et 1977). Etant donné que la transpiration et l'interception sont toutes deux fonction de l'évolution de l'indice foliaire, la simulation du développement du hêtre apparaît indispensable pour l'estimation de ces deux termes du bilan d'eau au printemps, dont dépendra bien entendu l'évolution du réservoir en eau du sol et la disponibilité de l'eau dans le sol en été.

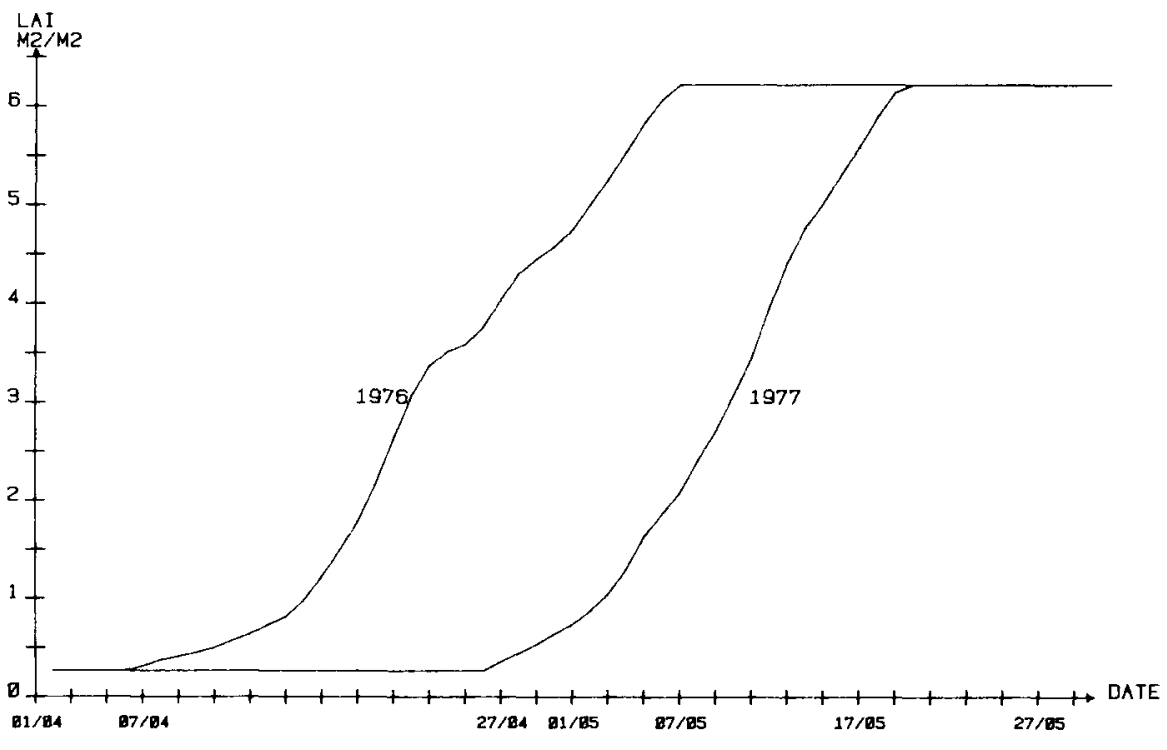

FiG. 1

Simulation de l'évolution de l'indice foliaire du hêtre (Fagus silvatica L.) au cours des printemps 1976 et 1977.

Simulation of the evolution of the leaf index of a beech tree (Fagus silvatica L.) during springs 1976 and 1977.

\subsection{Couvert de résineux}

La connaissance de la phénologie dans le cas de résineux semble moins cruciale pour l'estimation de l'évaporation que dans le cas d'un couvert de feuillus. D'une part il n’y a pas de cessation de la transpiration des résineux pendant l'hiver, d'autre part les fluctuations de l'indice foliaire, sans être nulles, sont nettement plus faibles pour les résineux que pour les feuillus. Aussi n’avons-nous donc pas élaboré de sous-modèle phénologique pour la simulation de l'évaporation du couvert de Douglas. On sait cependant que les aiguilles sont renouvelées tous les 3 à 4 ans. BEAdLE et al. (1982) ont noté dans une forêt de Pin sylvestre une variation de 7 p. 100 de l'indice foliaire au cours de l'année avec un maximum de juin à octobre. Aussenac (1979) a trouvé sous peuplement de Douglas à Nancy une chute des vieilles aiguilles en juillet-août, par mesure de la litière recueillie au sol. 


\section{Les paramètres internes de régulation du modèle}

Ce modèle utilise le concept de "résistance". Quatre résistances ont été définies : une résistance aérodynamique, deux résistances de structure et une résistance stomatique. Etant donné que ces résistances en série s'ajoutent pour constituer le frein global à l'évaporation du couvert (cf. partie 1) leurs modes de calcul et les ordres de grandeur de leurs valeurs sont importants à connaître.

\subsection{Résistance aérodynamique}

Celle-ci, fonction essentiellement du vent, est calculée en appliquant les lois relatives à la couche limite de surface, en condition de neutralité thermique, c'est-à-dire qu'aucune correction de stabilité/instabilité n'est irtroduite dans le modèle. En fonction du vent mesuré sur l'aéroport à 10 mètres de hauteur, elle est de la forme (CHASsAGNeuX \& ChOISNEl, 1986) :

$$
\mathrm{r}_{\mathrm{it}}=\frac{1}{\mathrm{k}^{2} \mathrm{Va}} \operatorname{Ln}\left(\frac{\mathrm{Zf}-\mathrm{d}}{\mathrm{Zof}}\right) \cdot \operatorname{Ln}\left(\frac{\mathrm{Za}}{\mathrm{Zoa}}\right) /\left(\frac{\mathrm{Zof}}{\mathrm{Zoa}}\right)^{11.117}
$$

En utilisant pour les paramètres hauteur de déplacement $\mathrm{d}$ et hauteur de rugosité $z_{o}$ les formules classiques $\left(z_{\mathrm{a}}=0,1 \mathrm{H}\right.$ et $\mathrm{d}=0.75 \mathrm{H}, \mathrm{H}$ étant la hauteur moyenne des arbres) nous obtenons pour la forêt de feuillus:

$$
r_{i:}=\frac{34,1}{V_{i 1}}
$$

Pour des vents variant de $1 \mathrm{~m} / \mathrm{s}$ à $10 \mathrm{~m} / \mathrm{s}$ elle variera donc de $34,1 \mathrm{~s} / \mathrm{m}$ à $3,4 \mathrm{~s} / \mathrm{m}$ (fig. 2). La résistance aérodynamique du couvert de résineux est calculée de façon similaire ; seule varie $H$, la hauteur du couvert. Les valeurs correspondant aux couverts sous lesquels les mesures expérimentales de sondages neutroniques ont été cffectuées sont les suivantes : $23 \mathrm{~m}$ pour les feuillus (Aussen.ac \& DuCrey, 1977) et $18 \mathrm{~m}$ pour les résineux (GRANIER, 1981).

\subsection{Résistances de structure}

Deux résistances de structure sont introduites dans le modèle. La première, notée $\mathrm{r}_{\text {sti }}^{*}$, concernant la réévaporation de l'eau interceptée a été ajustée à une fonction linéaire croissance de l'indice foliaire $\left(\mathrm{r}^{*}{ }_{\mathrm{tr}}=\mathrm{a}-\mathrm{b}\right.$ LAI/LAImax). Le couple de nombres a et $b$ a été optimisé de façon à minimiser l'erreur absolue annuelle et la somme des erreurs absolues mensuelles de la quantité d'eau nterceptée par le peuplement feuillu (cf. 4.1.). C'est cette résistance qui a été ajustée en premier lieu (Chassagneux, 1984). Le «meilleur» couple $(a, b)$ est $(20,70)$. Pour le peuplement de Douglas le peu de données disponibles concernant l'interception ne nous a pas permis de faire le même type d'ajustement. Faute de mieux la même fonction de résistance de structure a été adoptée pour le Douglas.

La résistance de structure pour la transpiration $\left(\mathrm{r}_{\mathrm{st}}\right)$ a été déterminée en second lieu, après avoir fixé l'expression de la résistance stomatique. Il est apparu que sa valeur est plus faible (rapport de 0.5 ) que la première résistance de structure introduite (Chassagneux \& Choisnel, 1986). 


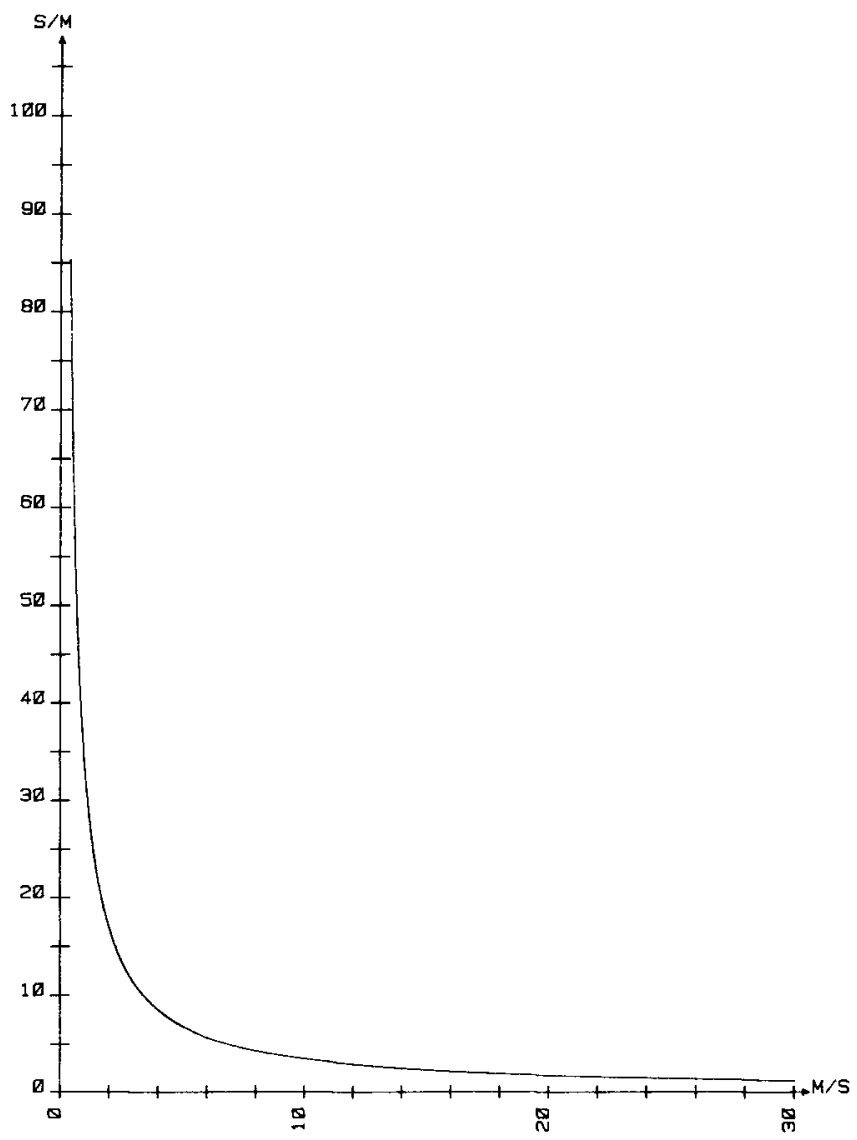

Fig. 2

Variation de la résistance aérodynamique calculée en fonction de la vitesse du vent mesurée à l'aéroport (à $10 \mathrm{~m}$ de hauteur au-dessus du sol), pour un couvert de feuillus.

Variation of the calculated aerodynamic resistance as a function of the wind speed measured at the airport (10 $\mathrm{m}$ height) for a hardwood canopy.

Cette résistance de structure $r^{*}{ }_{\text {str }}$ est un paramètre interne au modèle qui n'est pas strictement indépendant de la façon dont les variables météorologiques d'entrée sont prises en compte dans le calcul. En effet elle permet peut-être in fine de corriger indirectement une éventuelle mauvaise répartition de la pluie heure par heure. En effet si le modèle surestime le nombre réel d'heures de pluie, l'intensité moyenne de la pluie sera moindre et l'interception calculée sera trop importante. Rappelons à ce sujet que la pluie diurne ou nocturne (disponible par pas de 12 heures en station) est divisée par le nombre d'heures de pluie estimée par l'intermédiaire des codes "temps présent " relevés toutes les 3 heures, pour obtenir l'intensité horaire moyenne de la pluie. 


\subsection{Résistance stomatique (eau du sol non limitante)}

II s'agit d'une résistance stomatique globale du couvert pris dans son ensemble. Cette résistance stomatique est minimale en ce sens que l'eau du sol n'est pas un facteur limitant. Elle prend en compte les deux variables importantes pour la transpiration au niveau de la feuille: le rayonnement sous la forme du rayonnement solaire global reçu au sol, et le déficit de saturation en vapeur d'eau de l'air environnant (formule de type Lohammar) :

$$
r_{\text {sto }}=\left(\frac{R_{g}+R_{\mathrm{qu}}}{R_{g}}\right) \cdot\left[\frac{\alpha+\beta\left(e_{w}\left(T_{a}\right)-e\right)}{L A I}\right]
$$

Les paramètres de cette formule ont été ajustés dans l'ordre suivant : d'abord $\mathrm{R}_{\mathrm{go}}$, puis $\beta$ et enfin $\alpha$. Pour le paramètre $R_{\mathrm{g},}$ Halldin et al. (1979) indiquent qu'il correspond à la moitié du seuil de saturation lumineuse. La valeur retenue pour le couvert de feuillus $\left(110 \mathrm{~W} / \mathrm{m}^{2}\right)$ est légèrement inférieure à celle obtenue par SAugier et al. (1985) pour le chêne $\left(125 \mathrm{~W} / \mathrm{m}^{2}\right)$. Ensuite le paramètre $\beta$ a été ajusté, sachant que c'est le terme $\beta \cdot\left(\mathrm{e}_{\mathrm{w}}\left(\mathrm{T}_{\mathrm{a}}\right)-\mathrm{e}\right)$ qui fait le plus varier la résistance stomatique.

Cet ajustement a été nécessaire car les valeurs mentionnées dans la littérature (Saugier et al., 1985 ; Singh \& Szeicz, 1980; Tan \& Black, 1976 ; Stewart \& de Bruin, 1985) ne correspondent pas au même schéma d'analyse des processus physiques que notre présent modèle. En effet de nombreux auteurs utilisent plutôt le concept de résistance de surface englobant la résistance de structure, la résistance stomatique (eau non limitante) et la régulation du sol due à une limitation de la disponibilité en eau dans le sol. Les valeurs obtenues pour cette résistance globale du couvert sont difficilement comparables à des mesures effectuées sur des feuilles individuelles et pour des intervalles de temps courts. Il semble donc logique de considérer plutôt cette résistance stomatique comme un paramètre interne au modèle tout en sachant que la formulation employée tient compte des deux variables atmosphériques identifiées comme étant des facteurs de variation de cette régulation (rayonnement solaire et déficit de saturation de l'air). Les considérations précédentes font également ressortir le problème de la compatibilité des échelles de temps entre mesure et modèle pour toute comparaison chiffrée.

Les valeurs obtenues sont sensiblement différentes pour les deux couverts. Tout d'abord de fortes différences apparaissent pour des déficits de saturation élevés. Ainsi pour un rayonnement solaire global $R_{g}$ de $300 \mathrm{~W} / \mathrm{m}^{2}$ et un déficit de saturation de 15 hecto-Pascals, le couvert de résineux a une résistance supérieure de $20 \mathrm{~s} / \mathrm{m}$ à celle du couvert de feuillus. Ensuite on a dans les deux cas une diminution rapide de la résistance avec l'augmentation du rayonnement solaire reçu et cela quel que soit le déficit de saturation en vapeur d'eau observé. Enf n nous avons indiqué sur les figures 3 et 4 , respectivement pour un couvert de feuillus et un couvert de résineux, l'évolution de la résistance stomatique au cours d'une journée typique d'été avec un fort déficit de saturation de l'air l'après-midi. Ceci nous montre les réactions très différentes de deux couverts différents à un même climat. 


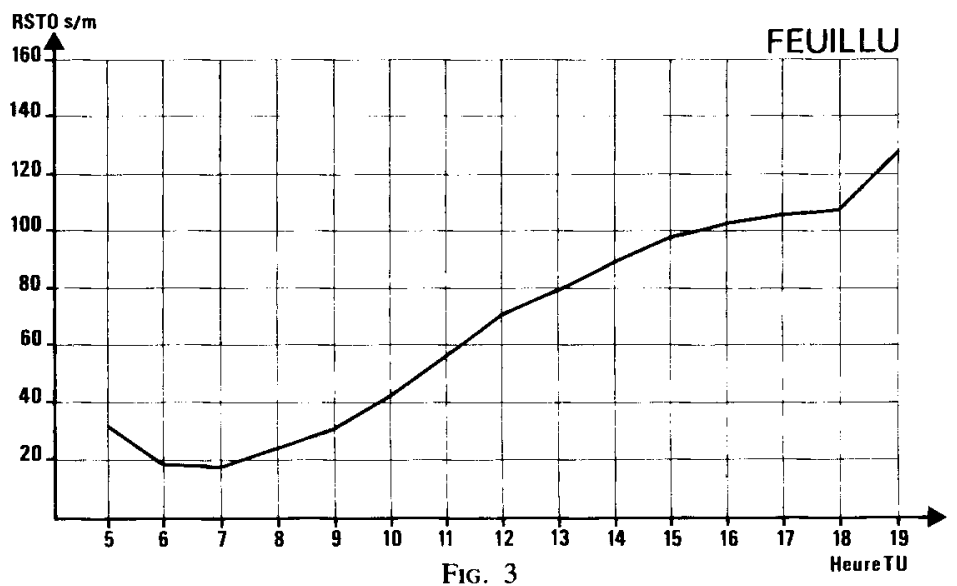

Evolution de la résistance stomatique minimale (eau du sol non limitante) au cours d'une journée ensoleillée pour un couvert de feuillus.

Evolution of the minimal stomatal resistance (water non limiting) during a sunny day for a hardwood canopy.

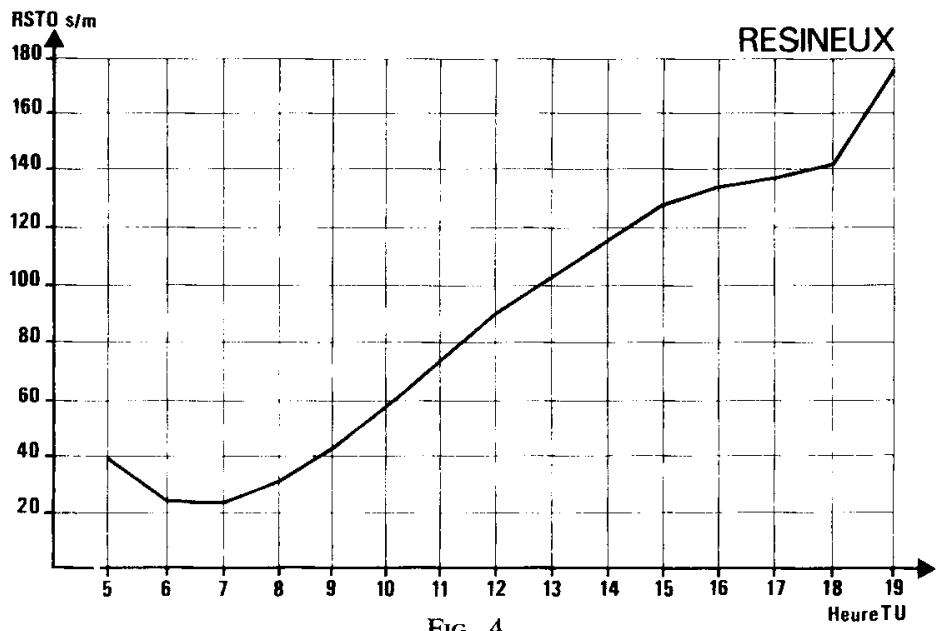

Evolution de la résistance stomatique minimale (eau du sol non limitante) au cours d'une journée ensoleillée pour un couvert de résineux.

Evolution of the minimal stomatal resistance (water non limiting) during a sunny day for a coniferous canopy.

\section{Simulation de l'interception}

\subsection{Peuplement de feuillus}

Celle-ci a été testée à partir de données de pluie au sol relevées par Aussenac (1968) au cours de l'année 1966-1967 sous le peuplement de hêtre. On connaît le 
rapport de la pluie mensuelle interceptée à la pluie mesurée sur le lieu même. L'interception calculée par le modèle a été comparée à une interception théorique correspondant au même rapport pluie au sol/pluie incidente mais avec pour pluie incidente celle mesurée à l'aéroport (fig. 5). L'erreur sur les mois d'été est de 8 p. 100 de la perte par interception (soit 2 p. 100 de la pluie incidente est mal répartie). Elle est de 14 p. 100 pour les mois d'hiver (soit 3 p. 100 de la pluie incidente). L'erreur est donc plus élevée en hiver. Cela est logique car d'une part l'interception d'un couvert défeuillé est mal connue en hiver, d'autre part le rôle de l'interception de la neige n'a pu être pris en compte. D’après AusSEnAC (1980) la capacité maximale de saturation est supérieure dans ce cas.

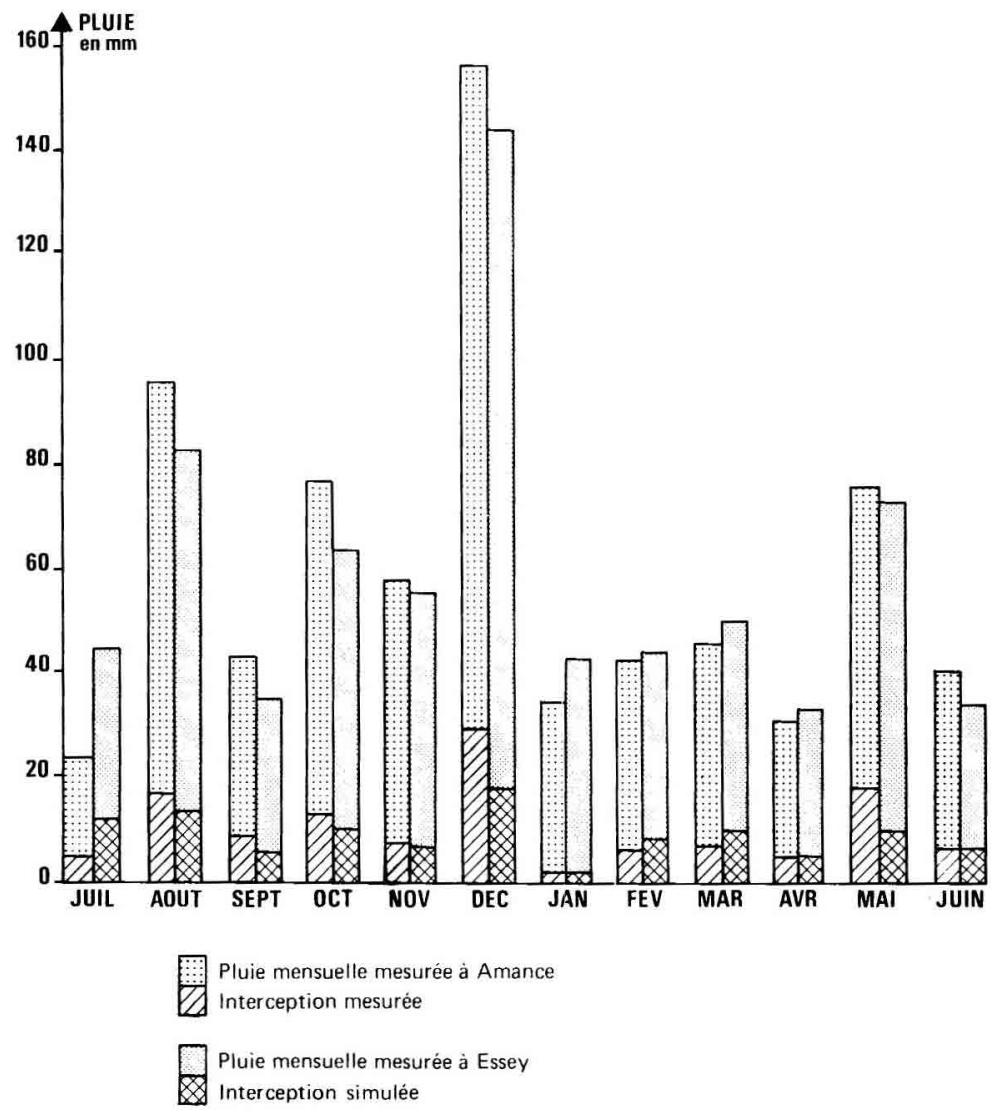

FIG. 5

Comparaison entre interception mesurée et interception calculée pour le peuplement de feuillus à Nancy-Amance (1966-1967).

Comparison between measured and calculated interception of the hardwood stand in Nancy-Amance (1966-1967). 


\subsection{Peuplement de douglas}

Seules quelques données correspondant à des périodes discontinues dans le temps étaient disponibles. Il semble que les résultats soient tout de même encourageants, l'erreur sur l'interception ne dépassant pas 10 p. 100 en été.

\section{Comparaison des résultats du modèle aux mesures de déstockage du sol}

\subsection{Définition du déstockage du sol et initialisation de la réserve en eau}

Le déstockage du sol est défini comme étant l'écart, en millimètres d'eau, entre le profil d'humidité volumique à la capacité au champ et le profil moyen à la date considérée (moyenne de 12 tubes individuels de sondage neutronique pour la parcelle feuillue, de 6 tubes pour la parcelle de Douglas) ; les mesures sont effectuées une fois par semaine pendant la période de végétation. Il est apparu pour l'été que lors de précipitations orageuses importantes il ne fallait pas comptabiliser la totalité de la pluie tombée pour tenir compte de phénomènes d'écoulement latéral par ruissellement superficiel. Divers essais nous ont amené à écrêter les pluies à une valeur seuil lorsque celle-ci était dépassée (20 mm en 12 heures, $30 \mathrm{~mm}$ en 24 heures).

La réserve a été initialisée à la valeur de $170 \mathrm{~mm}$, cette estimation paraissant justifiée par le fait qu'en 1976 le déstockage maximum correspondant à un des tubes a été de $177 \mathrm{~mm}$ sous peuplement feuillu sur 1,5 mètre de profondeur, le déstockage étant pratiquement nul en dessous de $70 \mathrm{~cm}$ de profondeur.

\subsection{Comparaison du déstockage calculé du sol aux mesures neutroniques}

Sur les figures suivantes $(6,7,8$ et 9) nous avons reporté l'évolution jour par jour du déstockage du sol calculé ainsi que le déstockage du sol déduit des mesures neutroniques de fréquence hebdomadaire pour les années 1976, 1977 et 1979 sous couvert de feuillus et pour l'année 1978 sous couvert de résineux. Sous chaque courbe est indiquée la différence de pluviométrie cumulée, entre deux dates de mesures neutroniques, entre celle mesurée en bordure de la forêt et celle mesurée à l'aéroport.

L'année 1976 (fig. 6) a été très intéressante car c'est elle qui a connu la plus forte «demande climatique " en évaporation du fait de la sécheresse et donc le déficit hydrique dans le sol le plus important. La dynamique du déstockage du sol est correctement simulée du mois de mai au mois de juillet. En août le déstockage calculé est supérieur à celui mesuré, malgré un écart de pluie d'une dizaine de millimètres au bénéfice de l'aéroport. Plusieurs explications peuvent être avancées: la sécheresse importante peut avoir entraîné une chute de feuilles précoce ce qui aurait diminué l'indice foliaire ; une perturbation fonctionnelle de la physiologie de l'arbre en ce qui concerne la transpiration peut avoir joué un rôle. Enfin malgré la présence de la couche de pseudogley imperméable des remontées capillaires ont pu se produire et ont déjà été mises en évidence sur ce type de substrat (Aussenac et al., 1984). La comparaison des résultats du modèle aux mesures de l'année 1976 ne constitue pas à proprement parler un test à part entière de la qualité du modèle car cette première 

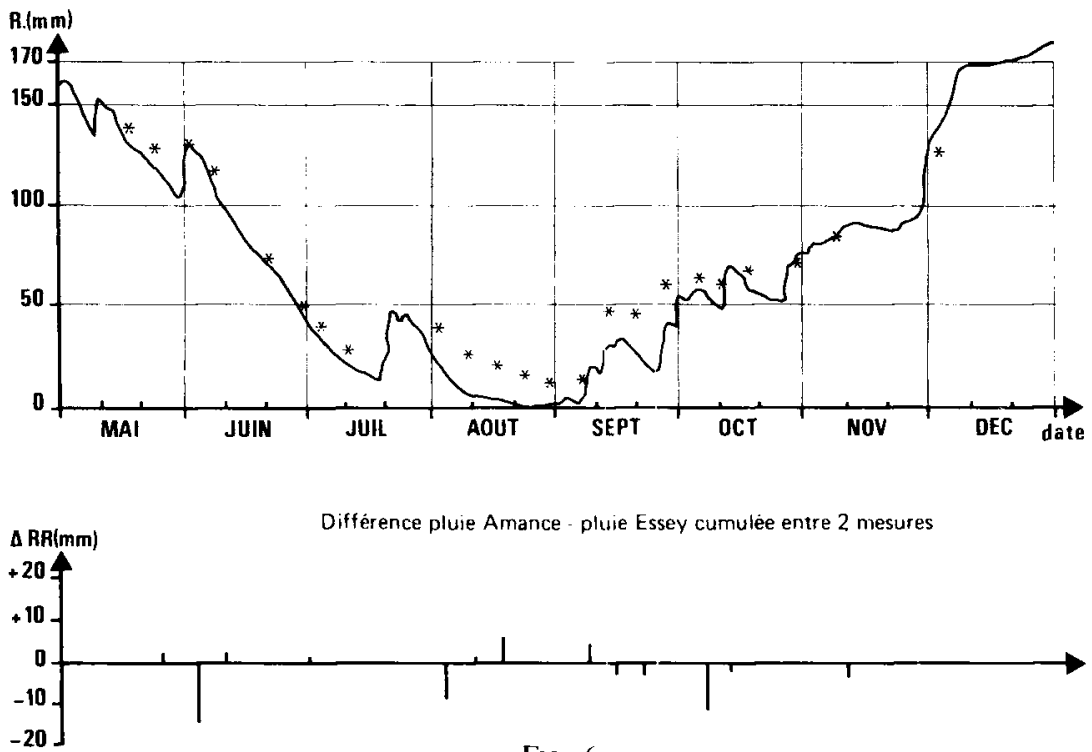

FIG. 6

Simulation du déstockage du sol (trait continu) sous couvert de feuillus en 1976 et comparaison aux valeurs expérimentales (*).

Simulation of the soil moisture deficit (continuous line) under the hardwood canopy in 1976 compared to experimental values (stars).
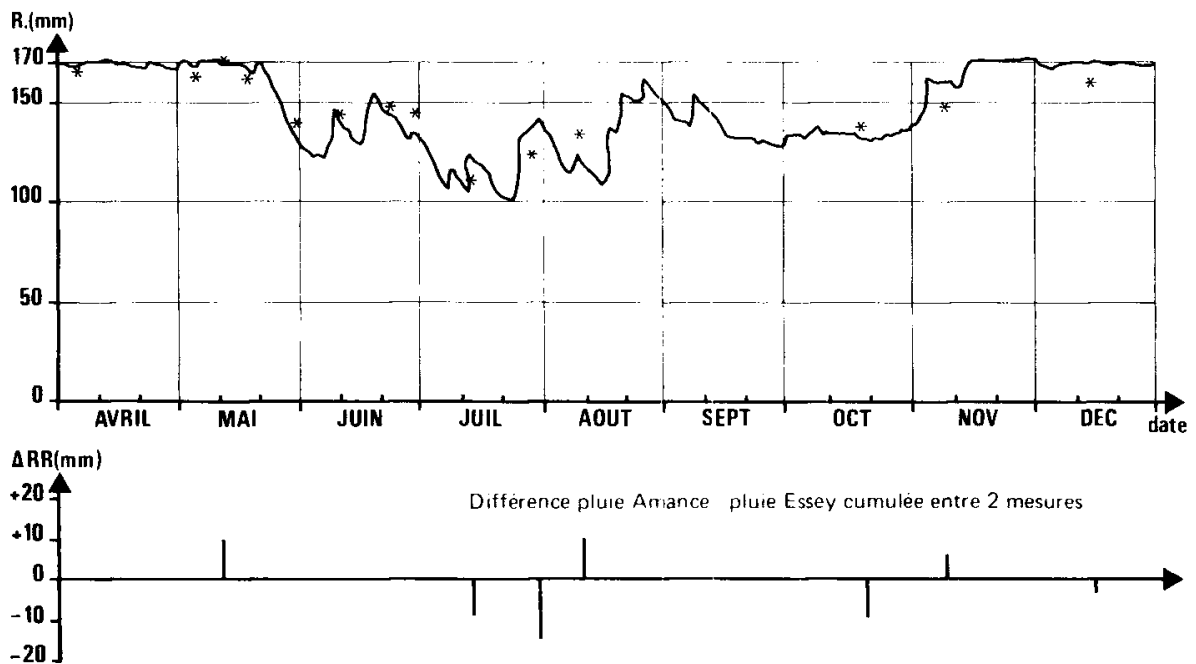

FiG. 7

Simulation du déstockage du sol (trait continu) sous couvert de feuillus en 1977 et comparaison aux valeurs expérimentales (*).

Simulation of the soil moisture deficit (cont. line) under the hardwood canopy in 1977 compared to experimental values (stars). 

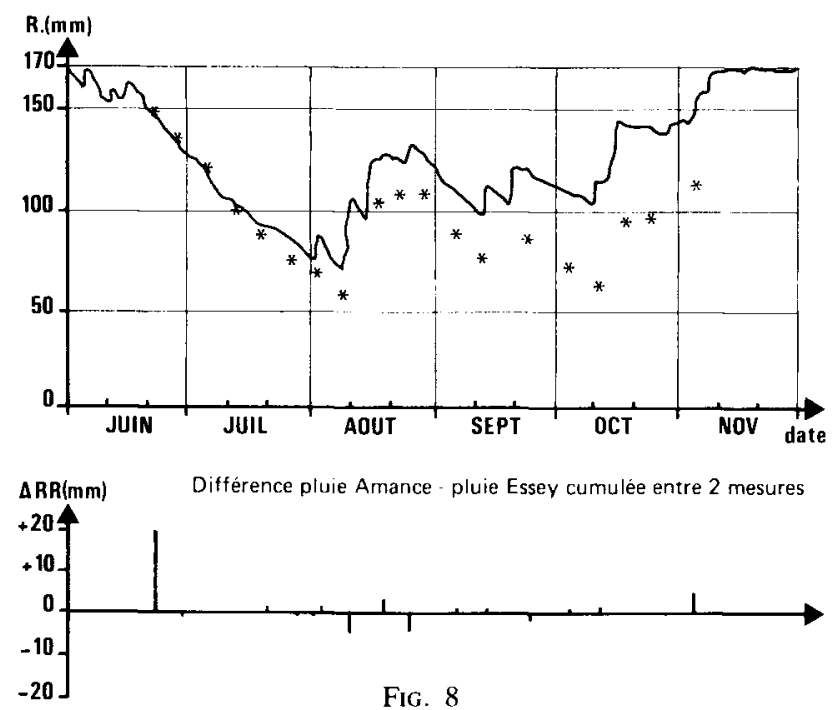

Simulation du déstockage du sol (trait continu) sous couvert de feuillus en 1979 et comparaison aux valeurs expérimentales (*).

Simulation of the soil moisture deficit (cont. line) under the hardwood canopy in 1979 compared to experimental values (stars).

année a servi à calibrer le modèle, en particulier la fonction de résistance stomatique (cf. 3.3.). Les résultats obtenus pour l'année 1977 (fig. 7) appellent peu de commentaire si ce n'est que du fait des fortes pluies le déstockage du sol est beaucoup plus limité, avec un maximum de $70 \mathrm{~mm}$ seulement fin juillet. La comparaison entre calcul et mesure est satisfaisante.

Les résultats de l'année 1979 sous couvert de feuillus (fig. 8) font apparaître un écart notable systématique à partir du 10 juillet. Il faut remarquer tout d'abord pour cette année que l'hiver précédent et le printemps ont été particulièrement pluvieux et le sol est encore à la capacité au champ au début du mois de juin. L'écart observé se crée essentiellement au cours du mois de juillet (le déstockage calculé reste inférieur au déstockage mesuré) et il est accentué en octobre pendant une semaine particulièrement pluvieuse ( $48 \mathrm{~mm}$ du 11 au 17 octobre), au cours de laquelle un certain ruissellement a dû se produire. Il semble que cette année exceptionnellement humide au printemps a créé des conditions hydriques très favorables à la croissance de l'indice foliaire, d'autant plus que la phase débourrement-feuillaison complète a coïncidé avec une période anormalement chaude (les températures moyennes des deuxième et troisième décades de mai et surtout de la première décade de juin 1979 sont chacune supérieures ou égales à la valeur du quatrième quintile de la période 1951-1980 à Nancy-Essey). La réalisation de cette phase a donc été très rapide (14 jours). Il est possible que la conjonction de conditions thermiques et hydriques favorables ait donné une augmentation de l'indice foliaire exceptionnelle et supérieure au maximum supposé $(6,2)$. Si tel est le cas, l'évapotranspiration réelle de l'arbre a été sous-estimée par le modèle.

La figure 9 présente l'évolution du déstockage calculé sous couvert de résineux au cours de l'année 1978. La différence qui apparaît en août et septembre s'explique par le 
TABleaU 1

Variabilité climatique comparée de l'évapotranspiration réelle $E T R$, de l'interception $E_{t}$ et de l'évaporation globale ET de cieux types de couverts

(cumul du 15 mai au 15 octobre, station de Nancy-Essey, période 1951-1980).

Compared climatic variability between two types of forest canopy of the actual evapotranspiration ETR, of interception $E_{i}$

and of the total evaporation ET (cumulated from May the 15th to October the 15th, Nancy-Essey, synoptic station, period 1951-1980).

\begin{tabular}{c|c|c|c|c|c|c|c}
\hline \hline & Mini & Année & $1^{\text {cr }}$ quartile & Médiane & $3^{c}$ quartile & Maxi & Année \\
\cline { 2 - 7 } ETR (mm) & & & & & & & \\
feuillus ... & 239 & 1954 & 270 & 288 & 299 & 318 & 1971 \\
résineux .. & 144 & 1954 & 210 & 222 & 237 & 265 & 1977 \\
E (mm) & & & & & & & \\
feuillus ... & 39 & 1959 & 56 & 60 & 66 & 78 & 1960 \\
résineux .. & 65 & 1976 & 98 & 109 & 115 & 131 & 1960 \\
ET (mm) & & & & & & & \\
feuillus ... & 302 & 1954 & 329 & 344 & 363 & 386 & 1971 \\
résincux .. & 255 & 1954 & 317 & 329 & 340 & 371 & 1977 \\
\hline
\end{tabular}

NB : Le terme E, correspond à l'cau interceptée et réćvapróe

(pluviométrie annuelle supérieure ou égale au décile supérieur) tandis que l'année 1971 obtenue pour les feuillus est caractérisée seulement par une pluviométrie estivale anormalement élevée (pluviométrie de l'ordre du quatrième quintile).

Enfin l'eau interceptée et évaporée est maximale pour les deux couverts en 1960 dont l'été est l'un des plus pluvieux de la période 1951-1980. Les valeurs minimales correspondent pour les résineux à l'année 1976 d'été exceptionnellement sec et pour les feuillus à l'année 1959 anormalement sèche en juillet uniquement.

\section{Applications et conclusion}

Les applications possibles d'un tel modèle sont multiples. Tout d'abord au niveau microclimatique il peut être utilisé pour suivre le bilan hydrique d'une parcelle expérimentale à l'aide de mesures micrométéorologiques au pas de temps horaire, ce qui permettrait de mieux caractériser l'effet de la réserve utile du sol et de la plus ou moins grande hétérogénéité du couvert sur son évaporation. A cette échelle de la parcelle certaines données de sortie du modèle telles que la réserve en eau du sol à une date donnée ou l'indice de stress hydrique décadaire pourraient servir d'indicateurs de production. Cette description au pas de temps décadaire plutôt que mensuel devrait permettre de mieux cerner les périodes de l'année importantes du point de vue 
disponibilité en eau pour le fonctionnement hydrique de l'arbre, voire de déterminer des valeurs seuils de la réserve en eau du sol séparément pour la croissance en hauteur et la croissance en diamètre (Aussenac et al., 1984).

Le modèle permet une évaluation séparée des diverses composantes du bilan hydrique du couvert. A cet égard il permet déjà une bonne répartition à faible échelle de temps de l'eau de pluie entre l'interception du feuillage et la pénétration dans le sol par une prise en compte correcte du régime pluviométrique sous les aspects d'intensité des épisodes pluvieux, de répartition des épisodes pluvieux dans le temps, et du niveau d'évaporation potentielle pour l'interception et la transpiration. A une échelle temporelle plus large il s'agit également de faire la part du rôle respectif des régimes pluviométriques automnal, hivernal, printanier et estival dans l'évaporation du couvert et la croissance annuelle, ce qui peut permettre de mettre en évidence des arrière-effets d'une sécheresse ou l'effet des conditions hydriques de l'année précédente.

La simulation du bilan hydrique d'un couvert forestier sur une longue série climatique apporte des éléments de décision pour le choix de telle ou telle espèce la mieux adaptée sur le plan de la production au régime pluviométrique régional. Sur le plan hydrologique les périodes à excédent pluviométrique et la nécessité éventuelle du drainage sont importantes à connaître. Il reste enfin un certain nombre de questions en suspens pour l'utilisation future du modèle : test dans des climats de régime pluviométrique différent, notamment en région méditerranéenne, mieux connaitre les phénomènes d'interception et d'évaporation en période hivernale, notamment pour les résineux. Alors les potentialités régionales de production forestière pourront être reliées à la disponibilité en eau.

\section{Summary \\ Modelling the total evaporation of a forest canopy. II. - Calibrations and results of the model}

The second part of this article presents the comparison between the model's outputs and weekly measurements of the soil moisture deficit inferred from volumetric moisture profiles. These measurements made at the National Forest Research Center in Nancy span over four years : three years (1976, 1977 and 1979) under a hardwood canopy (Fagus silvatica L.) and one year (1978) under a coniferous canopy (Pseudotsuga menziesii)

The different steps in the calibration of the model, namely the determination of the parameters in the parameterization equations, are introduced first. The statistics over a long period (33 years at Nancy's site) of the ecophysiological variables, such as the date of budding and the date of full leaf growth of beech trees, are indicated in paragraph 3.1. Figure 1 shows how the growth period of the leaf area index (from budding to maximum L.A.I.) can be delayed from one year (1976) to another (1977), depending on their specific spring climate. The different resistances included in the regulation of the evapotranspiration have been adjusted (paragraph 4 , equation 1 to 3 and figures 2 to 4 ).

The results obtained with the interception sub-model show a relative error of 8 p. 100 in summer and 14 p. 100 in winter regarding the rain intercepted by the beech stand over a 12-month measurement period (fig. 5). Then the calculated soil moisture deficit by the model is compared to the one that had been measured. The maximum available water has been set to the value of 170 millimeters, a value close to the maximum observed deficit in 1976. The results obtained for the first year (1976, a year of exceptional spring and summer drought) give a correct evaluation of the soil drying from May to July (fig. 6). The latter year measurements helped to calibrate some of 
the parameters of the model, particularly the stomatal resistance function. The comparison between calculations and measurements is also satisfactory for the following year, 1977, a year marked by a limited soil moisture deficit due to heavy rains, the deficit reaching only $70 \mathrm{~mm}$ in late July (fig. 7). The third simulated year (1979) for the hardwood stand exhibits a systematic deviation from mid-July onwards (fig. 8). This deviation corresponds to an underestimation of the tree's actual evapotranspiration. It would seem that the year 1979, with an abnormally rainy spring, could be associated with favourable soil water conditions and high air temperatures during the late in the season, but limited phase of increasing leaf area index. Lastly one year (1978) of simulation for the Douglas tree canopy is presented. Marked by an autumnal drought it shows, after a deviation during a period following thunderstorms, a correct evaluation of the final soil moisture deficit towards its end.

Then the use of the model's output data (actual evapotranspiration of the forest canopy, ratio of the soil water reserve to its maximum value) is discussed. An index of water stress calculated from two output variables of the model is proposed (equation 4).

Such a model also enables us to simulate the total evaporation and the soil water balance of a forest canopy over a long climatic period. This has been done using Nancy's synoptic station data recorded over 32 years (1950-1981). Statistics were performed regarding interception (intercepted and reevaporated water), the actual evapotranspiration and total evaporation calculated from the 15th of May to the 15th of October, which is the mean period of complete foliage for the hardwood stand. The calculated evapotranspiration of the beech tree canopy is 20 p. 100 in excess of that of the Douglas tree canopy. On the other hand, because of their higher canopy storage capacity, interception of rain by the Douglas tree is greater than the one by beech trees. Nevertheless, the hardwood canopy's total evaporation remains greater than the one of Douglas trees over the above mentioned 5-month period.

Testing the model in climates with a different rain regime, for example in a mediterranean climate, remains to be done. Also the phenomena of interception and evaporation should be more investigated during the winter season.

Key words : Coniferous, evaporation, evapotranspiration, hardwood, interception, model, resistance, soil moisture deficit.

\section{Liste des symboles utilisés}






\section{Références bibliographiques}

Aussenac G., 1968. Interception des précipitations par le couvert forestier. Ann. Sci. for., 25 (3), 135-156.

Aussenac G., Ducrey M., 1977. Etude bioclimatique d'une futaie feuillue de l'Est de la France. I. Analyse des profils microclimatiques et des caractéristiques anatomiques et morphologiques de l'appareil foliaire. Ann. Sci. for., 34 (4), 265-284.

Aussenac G., 1979. Production de litière dans quatre jeunes peuplements de Douglas dans l'Est de la France. Rev. for. fr., 31, 1, 15-19.

Aussenac G., Granier A., 1979. Etude bioclimatique d'une futaie feuillue (Fagus silvatica L. et Quercus sessiliflora Salisb.) de l'Est de la France. II. Etude de l'humidité du sol et de l'évapotranspiration réelle. Ann. Sci. for., 36 (4), 265-280.

Aussenac G., 1980. Le cycle hydrologique en forêt. In Pesson P., Actualités d'éculogie forestière, Gauthier-Villars/Bordas, Paris, 283-307.

Aussenac G., Bollangeat C., 1980. Interception des précipitations et évapotranspiration réclle dans les peuplements de feuillus (Fagus silvatica L.) et de résineux (Pseudotsuga menziesii (Mirb.) Franco). Ann. Sci. for., 37 (2), 91-107.

Aussenac G., Granier A., Ibrahim M., 1984. Influence du dessèchement du sol sur le fonctionnement hydrique et la croissance du Douglas (Pseudotsuga menziesii (Mirb.) Franco). Acta Oecol. Plant. 5 (19), 3, 241-253.

Beadle C.L., Talbot H., Jarvis P.G., 1982. Canopy structure and leaf area index in a mature Scots pine forest. Forestry, 55, 2, 105-123.

BLACK T.A., 1979. Evapotranspiration from Douglas fir stands exposed to soil water deficits. Water Resour. Res., 15 (1), 164-170.

Chassagnfux P.. 1984. Etude et modélisation de l'evapotranspiration globale et du bilan hydrique d'un couvert forestier. Rapport de stage de recherche de l'Ecole Nationale de la Météorologie, Subdivision d’Agrométéorologie, Paris, $84 \mathrm{p}$.

Chassagneux P., Choisnel. E., 1986. Modélisation de l'évaporation globale d'un couvert forestier I. Principes physiques et conception du modèle. Ann. Sci. for., 43 (4), 505-520.

Cholsnel E., 1985. Un modèle agrométéorologique opérationnel de bilan hydrique utilisant des données climatiques. Comm. Conf. Int. CIID, "Les besoins en eau des cultures", Paris, éd. INRA, 115-132.

Granier A., 1981. Etude des relations entre la section du bois d'aubicr et la masse foliaire chez lo Douglas [Pseudotsuga menziesii (Mirb.) Franco]. Ann. Sci. for., 38 (4), 503-512.

Halidin S., Gripp H., Pertru K., 1979. Model for energy exchange of a pine forest canopy. International society for Ecological Modelling, Copenhagen. In Comparison of forest water and energy exchange models, S. Halldin ed., Elsevier, Amsterdam, 59-75.

Nizinski G., 1986. La modélisation du bilan de l'eau d'une futaie de chêne en forêt de Fontainebleau. Thèse de Docteur-Ingénieur. Université d'Orsay, $101 \mathrm{p}$.

Saugier B., Halldin S., Pontailler J.Y., Nizinski G., 1985. Bilan hydrique des forêts de chêne et de hêtre à Fontainebleau. Mesures et modélisation. Rev. Palais Déc., 13, 130, 187-200.

Singh B, SZeicZ G., 1980. Predicting the canopy resistance of a mixed hardwood forest. Agric. Meteor., 21, 49-58.

Stewart J.B., De Bruin H.A.R., 1985. Preliminary study of dependence of surface conductance of Thetford forest on environmental conditions. In The forest-atmosphere interaction, D. Reidel Publ. Co., 91-104.

TAN C.S., BLACK T.A., 1976. Factors affecting the canopy resistance of a douglas pine forest. Bound. Layer Meteor., 10, 475-488. 